\title{
Editorial: UV Astronomy 2011
}

\author{
Boris Shustov • Ana I. Gómez de Castro • \\ Mikhail Sachkov • Michael A. Dopita
}

Received: 24 May 2011 / Accepted: 31 May 2011 / Published online: 17 June 2011

(C) Springer Science+Business Media B.V. 2011

\section{Keywords UV Astronomy}

In the past few years space UV astronomy has been seeing somewhat of a renewal, thanks to new facilities coming on line, and others reaching an advanced stage of development. Consequently, we are pleased to present this Special Issue of Astrophysics \& Space Science; “UV Astronomy 2011”. This builds on the remarkable progress made in the field since our earlier Special issue: "Space Astronomy: the UV window to the Universe" which appeared in 2008.

The UV spectral range is of prime interest for astrophysics since it is here that we find the resonance lines of the most abundant atoms and ions in plasmas with temperatures between $3000 \mathrm{~K}$ and $300,000 \mathrm{~K}$ together with the electronic transitions of the most abundant molecules $-\mathrm{H}_{2}, \mathrm{CO}$, $\mathrm{OH}, \mathrm{CS}, \mathrm{S}_{2}, \mathrm{CO}_{2}^{+}, \mathrm{C}_{2}, \mathrm{O}_{2}$ and a number of other species.

\section{B. Shustov · M. Sachkov}

Institute of Astronomy, Russian Academy of Sciences,

Pyatnitskaya 48, 119017 Moscow, Russia

B. Shustov

e-mail: bshustov@inasan.ru

A.I. Gómez de Castro $(\varangle)$

Fac. de CC. Matemáticas, Universidad Complutense de Madrid, Plaza de Ciencias 3, 28040 Madrid, Spain

e-mail: aig@mat.ucm.es

M.A. Dopita

Institute for Astronomy, University of Hawaii, 2680 Woodlawn

Drive, Honolulu, HI 96822, USA

M.A. Dopita

Research School of Astronomy and Astrophysics, Australian

National University, Cotter Rd., Weston ACT 2611, Australia
Moreover, the UV radiation field is both a powerful photoionizing and an astrochemical agent. Unfortunately for astronomers (but perhaps fortunately from the point of view of our life form!), the UV range is not accessible from the ground. Progress in the field therefore depends upon a steady supply of space-based instrumentation of increasing power and complexity.

Ultraviolet spectroscopy provides some of the most fundamental diagnostic data to discern the physical characteristics of planets, stars, galaxies and interstellar and intergalactic matter. The UV also allows access to Lyman- $\alpha$ at low to moderate redshifts, high ionization stages of key diagnostic elements and unique abundance information for both atomic and molecular species that cannot be obtained at other wavelengths. The O VI, C IV, N V lines are essential to trace the evolution of the intergalactic gas that is too cool to emit $\mathrm{X}$ rays. High-resolution UV spectroscopy is essential to study the dynamics and composition of interstellar gas. Such data are essential to draw a complete picture of the Universe.

The energy distribution of hot stars (those earlier than Atype) peaks at UV wavelengths. We can therefore observe the very important massive main sequence stars that are responsible of the chemical enrichment of the Universe and which act as dominant sources of radiant energy into the diffuse medium. UV observations also allow us to isolate the contribution of hot components in cool environments such as stellar chromospheres in cool stars, hot stars or the Active Galactic Nuclei (AGN) in Elliptical galaxies and massive spirals.

Above all, UV astronomy is a fundamental tool to allow us to study in depth the local Universe, understood as the Universe up to $z=1.8-2$. This redshift span roughly covers $80 \%$ of cosmic time! Furthermore, UV astronomy informs the interpretation of objects seen at much higher redshifts for which their UV emissions have been redshifted into the 
optical or IR domain. The data obtained from the high redshift universe with the current generation of large opticalinfrared telescopes cannot be understood without first having a proper interpretation of the nearby analogs of these distant objects.

For these reasons, UV astronomy remains a very active area of astrophysical research, and has been for half a century. The number of short term experiments and long term space observatories designed for observation in UV domain is large-it now exceeds 60 . The most powerful observatory ever flown, the Hubble Space Telescope (HST) is expected to operate till 2014-2016. In 2014, the World Space Observatory-Ultraviolet (WSO-UV) mission will be flown. This will guarantee access to the UV Universe till 2024. Hopefully, both projects HST and WSO-UV will coexist for sometime.

The Cosmic Origins Spectrograph (COS) is now fully operational on board HST, providing superb results. The Wide Field Camera 3 (WFC3), installed at the same time as COS allows high quality imaging in the near-UV, at the same time providing comparable image quality through the optical and into the near-IR wavelengths. The World Space Observatory-UltraViolet (WSO-UV) is progressing; the Spectrographs have ended Phase B, and the design of the imaging instrument has changed into the Imaging and Slitless Spectroscopy Instrument for Surveys (ISSIS), after Italy withdrew the project in 2008, and Spain took over the development of an imaging instrument for the WSO-UV. Finally, the first astronomical images obtained with the Fresnel Interferometers have been obtained. Making use of the long tube of the Nice Observatory, amazing images of Mars satellites and double stars have been obtained to probe successfully the technology. Fresnel interferometers look like the "solution" for large collecting apertures in space for the next decade. An extensive report on the current status of technological development of these devices can be found in a dedicated volume to Fresnel Interferometers, currently in press by Experimental Astronomy.

Some amazing scientific results are also presented in this volume as the report, by the HST/COS team (see the France et al. contribution), of the UV observations of the transiting extrasolar planetary system HD209458b. Also, the current status of the GALactic Evolution eXplorer (GALEX) survey of the UV sky is presented.

This volume covers four main topics: [1] stellar physics and evolution, [2] planets, [3] Milky Way and other galaxies and finally, [4] UV Instrumentation with an update on the current status of the main missions and technologies.

Many of the science articles here help to define the core science mission of WSO-UV. For this reason, this volume contains an unusually great collection of Russian astronomical research and, to a lesser degree, of Spanish and German astronomy, who are the partners in the WSO-UV project together with the Ukraine. As Editors, we have tried to ensure that the English writing was as good as possible, and we hope we have succeeded.

Acknowledgements We acknowledge the support of the Russian Academy of Sciences, the Federal Space Agency of Russia and the Network for UV astrophysics (NUVA), which are making possible the WSO-UV mission. 